\title{
The Perceived Personal Control (PPC) Questionnaire: Reliability and Validity in a Sample From the United Kingdom
}

\author{
${ }^{1}$ School of Biomedicine, University of Manchester, Manchester, UK \\ ${ }^{2}$ School of Psychological Sciences, University of Manchester, Manchester, UK \\ ${ }^{3}$ School of Community-Based Medicine, University of Manchester, Manchester, UK \\ ${ }^{4}$ Department of Psychology, Tel Aviv University, Tel Aviv, Israel \\ ${ }^{5}$ School of Nursing, Midwifery \& Social Work, University of Manchester, Manchester, UK
}

Marion McAllister, ${ }^{1 *}$ Alex M. Wood, ${ }^{2}$ Graham Dunn, ${ }^{3}$ Shoshana Shiloh, ${ }^{4}$ and Chris Todd ${ }^{5}$

Received 21 February 2011; Accepted 17 October 2011

Outcome measures are important assessment tools to evaluate clinical genetics services. Research suggests that perceived personal control (PPC) is an outcome valued by clinical genetics patients and clinicians. The PPC scale was developed in Hebrew to capture three dimensions of PPC: Cognitive, decisional, and behavioral control. This article reports on the first psychometric validation of the English translation of the PPC scale. Previous research has shown that the Hebrew and Dutch translations have good psychometric properties. However, the psychometric properties of the English translation have not been tested, and there is disagreement about the factor structure, with implications for how to score the measure. A total of 395 patients attending a clinical genetics appointment in the United Kingdom completed several measures at baseline, and a further 241 also completed measures at $2-4$ weeks follow-up. The English language PPC has (a) a one-factor structure, (b) convergent validity with internal health locus of control (IHLC), satisfaction with life (SWL), depression, and authenticity, (c) high internal consistency ( $\alpha=0.83$ ), and (d) sensitivity to change, being able to identify moderate changes in PPC following clinic attendance (Cohen's $\mathrm{d}=0.40$ ). These properties suggest the English language PPC measure is a useful tool for both clinical genetics research and for use as a Patient Reported Outcome Measure (PROM) in service evaluation. $\odot 2011$ Wiley Periodicals, Inc.

Key words: medical genetics; patient reported outcome measure; psychometrics; questionnaires; perceived personal control; genetic counseling

\section{INTRODUCTION}

Measuring patient outcomes from clinical genetics services is important to enable (a) different services to be compared in clinical practice and (b) evaluation of new interventions in research [McAllister, 2010]. Patient outcomes are usually measured using
How to Cite this Article:

McAllister M, Wood AM, Dunn G, Shiloh S, Todd C. 2011. The perceived personal control (PPC) questionnaire: Reliability and validity in a sample from the United Kingdom.

Am J Med Genet Part A .

a combination of clinical outcomes and Patient Reported Outcome Measures (PROMs). PROMs are short self-completion questionnaires, often designed to capture psychological "constructs", such as anxiety and/or depression.

Recent research has identified that perceived personal control (PPC) is a patient outcome that is valued by both patients and genetics clinicians in the United Kingdom (UK) [Payne et al., 2007]. PPC is a construct reflecting the degree to which a person believes that a situation is under their control, and that she or he is able to bring about a desirable change to the situation or avoid an undesirable situation. PPC has been conceptualized in a number of different ways [Skinner, 1996]. A tripartite structure of PPC [Averill, 1973] has been usefully applied to genetic counseling [Shiloh et al., 1997], and includes three dimensions: Behavioral, decisional, and cognitive control. Behavioral control refers to the belief that there is action that can be taken to change a situation;

${ }^{*}$ Correspondence to:

Dr. Marion McAllister, BA(Mod), MSc, PhD, Institute of Medical Genetics, Cardiff University, Heath Park, Cardiff CF14 4XN, United Kingdom.

E-mail: mcallistermf@cardiff.ac.uk

Published online 00 Month 2011 in Wiley Online Library

(wileyonlinelibrary.com).

DOI 10.1002/ajmg.a.34374 
decisional control refers to the belief that there are different courses of action that can be chosen to change a situation; and cognitive control refers to understanding and finding meaning in a situation, as well as using patterns of thinking that can reduce the stressfulness of a situation. This article reports on the first test of the psychometric properties of the English translation of the PPC scale, which was developed to operationalize this construct as a PROM for genetic counseling services.

The PPC was originally developed and validated in Hebrew by a team in Israel [Berkenstadt et al., 1999], who also provided an English translation of the measure in the scale development paper, which was published in the American Journal of Medical Genetics. The English translation from Hebrew was checked for accuracy by a back translation procedure. A few mismatches were corrected after discussion. The PPC is a nine-item scale, with items presented as statements (e.g., "I feel I can make decisions that will change my family's future") to which respondents can indicate their degree of agreement, with three response categories ("do not agree", "somewhat agree", "completely agree"). Validation of the Hebrew PPC with an Israeli reproductive genetic counseling sample $(\mathrm{n}=256)$ suggested good psychometric properties including (1) sensitivity to change over time, (2) convergent validity with knowledge, satisfaction, counseling evaluations, and expectation fulfillment, and (3) good internal consistency (Cronbach's $\alpha=0.83-0.86)$. Validation of a subsequent Dutch translation [Smets et al., 2006] also suggested (1) sensitivity to change, (2) convergent validity with patient satisfaction, and (3) good internal consistency (Cronbach's $\alpha=0.79-0.81$ ).

The English and Dutch translations of the PPC have been used in evaluations of clinical genetics services [e.g., Davey et al., 2005; Pieterse et al., 2011], demonstrating growing acceptability of the PPC by the research community. However, the psychometric properties of the English translation have never been reported. In addition, there is disagreement about the factor structure of the scale, leading to confusion about whether to use an overall (PPC) score, or whether to form additional sub-scales. Confirmatory factor analysis (CFA) on the Hebrew PPC found that a three-factor solution, corresponding to behavioral, decisional, and cognitive control, provided a good fit for the Israeli data [Berkenstadt et al., 1999]. However, since exploratory factor analysis (EFA) was not performed, and as this CFA solution was never compared to any other potential solutions, it is possible that another (better fitting) structure actually exists. Supporting this possibility, EFA of the Dutch translation failed to support the three-factor solution, and the authors conclude that only one factor underlies the data [Smets et al., 2006].

The present study tests the psychometric properties of the English translation of the PPC [Berkenstadt et al., 1999] in a sample of patients using a clinical genetics service in the UK. The specific aims were to (1) explore the factor structure of the English translation of the PPC using robust exploratory factor analytic methods, (2) to assess concurrent validity with health locus of control, anxiety, depression, satisfaction with life (SWL), authenticity, and satisfaction with genetic counseling (SWGC), (3) to assess sensitivity to change pre- and post-genetic counseling, and (4) test internal consistency. PPC responses were expected to correlate with other constructs as follows:

\section{Convergent Validity}

- PPC scores will correlate positively with Internal Health Locus of Control (IHLC) because both constructs relate to feelings of control in a health context; however, the correlation was not expected to be high, because PPC relates to control in a more general sense than IHLC.

- PPC will correlate positively with SWL, because personal control reflects good psychological functioning, and this will be reflected in associated increased levels of life satisfaction. Once again, the correlation was expected to be moderate, since the constructs are related, but not the same.

- PPC scores will correlate negatively with anxiety and depression, because personal control reflects good psychological functioning [Skinner, 1996] (low anxiety and depression). These correlations were expected to be moderate, since the constructs are related, but not the same.

- PPC will correlate positively with authenticity, which is a construct based on Carl Rogers person-centered concept. If genetic counseling could be said to be informed by any theoretical framework, it is the person-centered approach. Authenticity was expected to correlate positively with PPC, as both concepts reflect aspects of self-determination. However, the size of the correlation was expected to be small, because Authenticity reflects self-determination in a very general sense, and PPC reflects self-determination in a very specific context (genetic counseling).

- Change in PPC scores after attendance at a genetics clinic will correlate positively with SWGC scores, reflecting the hypothesis that patients value PPC as an outcome from genetic counseling. The size of the correlation was expected to be moderate, based on previously published correlations [Berkenstadt et al., 1999].

\section{Discriminant Validity}

- PPC scores will not correlate significantly with Powerful Others Health Locus of Control (PHLC) because PPC does not include beliefs about the control held by other people over one's health, as PHLC does.

- PPC scores will not correlate significantly with Fate Health Locus of Control (FHLC) because PPC does not include beliefs about health being determined by fate, as FHLC does.

\section{Known Groups Validity/Sensitivity to Change}

- Known group validity requires a strong a priori expectation that groups differ on the construct. There is insufficient previous research with clinical genetics samples to develop strong a priori expectations about differences in PPC levels between different sub-groups of patients. However, there was the expectation that the sample as a whole would have higher PPC levels post-clinic, which makes a pre- and post-clinic comparison test both a test of sensitivity to change and of known group validity. 


\section{METHODS}

\section{Participants}

This study was conducted with a sample of patients attending the Genetic Medicine department based at Central Manchester University Hospitals NHS Foundation Trust (CMFT). All new patients referred to Genetic Medicine between February 2010 and July 2010, except those referred to the genetic urgency clinic (usually patients with a current potentially at-risk pregnancy) were invited to participate. Parents referred to the urgency clinic were excluded because the time between referral and appointment is less than a week and it would have been logistically difficult to recruit them prior to their clinic appointment. In addition, there were concerns that these patients may have been distressed by the added burden of completing research questionnaires at this time. Patients were recruited by a letter from a member of the clinical team, enclosing an Information Sheet, and a questionnaire pack.

\section{Instruments and Data Collection}

The questionnaire pack included the English translation of the PPC, and other questionnaires to assess construct (concurrent) validity, and a prepaid envelope for return of the completed questionnaires. There is no gold standard measure of PPC in English, and so the following additional questionnaires were selected based on expected relationships with PPC: (i) The Multidimensional Health Locus of Control Scales (MHLC) [Wallston et al., 1978], (ii) the State-Trait Anxiety Inventory short form (STAI-6) [Marteau and Bekker, 1992], (iii) the Personal Health Questionnaire (PHQ-9) (a measure of depression) [Gilbody et al., 2007], (iv) the SWL questionnaire [Pavot and Diener, 1993], (v) the Authenticity scale [Wood et al., 2008], and (vi) the short form of the SWGC questionnaire [Shiloh et al., 1990]. Participating patients were asked to complete the PPC and questionnaires (i) $-(\mathrm{v})$ prior to their first appointment in Genetic Medicine. Participants who indicated on the first questionnaire pack that they were happy to receive a second questionnaire pack after their appointment were sent the PPC questionnaire along with questionnaires (i)-(vi) about 4 weeks after their first appointment.

Data were collected on (1) type of referral, e.g., cancer, neuromuscular, learning disability, etc. and (2) what was provided to the patient in clinic, e.g., a diagnosis, a risk figure, new information, screening recommendations/referral, a genetic test, etc. Ethical review was conducted by the Central Manchester NHS Research Ethics Committee (ref. 09/H1008/111).

\section{Data Treatment and Analyses}

Data were entered into SPSS for Windows 16.0 (IBM). For questionnaires with minimal missing data $(<20-25 \%$, depending on item numbers), prorating was used to "impute" missing values [Morriss and Coyle, 1994]. Questionnaires with more than 20-25\% missing values (13 questionnaires) were excluded from the analysis. PPC responses were analyzed using EFA, rather than CFA, to allow for the possibility that the structure of PPC predicted by Averill (i.e., three factors: Cognitive, decisional, and behavioral control) may not exist in the data. EFA was conducted using maximum likelihood, and parallel analysis was used to identify the number of factors to extract. Parallel analysis was used because it has been shown to be a more robust and reliable method for determining the number of factors in a dataset than either (1) the number of factors theoretically expected, the method used by the Israeli group, or (2) the eigenvalues greater than one rule, used by the Dutch group [Zwick and Velicer, 1986; Fabrigar et al., 1999]. Internal consistency was assessed using Cronbach's alpha, and sensitivity to change over time was analyzed by calculating an intra-class correlation using analysis of variance. Concurrent validity was assessed using bivariate correlation.

\section{RESULTS}

In total, 395/1,205 (32.8\%) of patients attending the Genetic Medicine department at St. Mary's Hospital in Manchester completed a baseline questionnaire pack prior to their first appointment. Of these, 374/395 (94.7\%) attended their appointment, and a further $241(64.4 \%)$ completed a post-clinic questionnaire pack 2-4 weeks after their appointment. Respondents were predominantly female $(79.5 \%)$ and White British $(89.6 \%)$. Of note, there were no significant differences in gender, ethnicity, condition type, or reason for referral between the samples responding at (a) preclinic only and (b) both pre- and post-clinic. However, participants responding both pre- and post-clinic were significantly older than those responding at baseline only. Sample characteristics are reported in detail elsewhere [McAllister et al., 2011].

The post-counseling data were subjected to EFA. The eigenvalues (and associated \% of variances) were $3.8(43.1 \%), 1.22(13.6 \%)$, 0.85 (9.4\%), 0.74 (8.3\%), 0.63 (7.0\%), 0.53 (5.9\%), 0.51 (5.7\%), $0.34(3.7 \%)$, and $0.29(3.2 \%)$. These eigenvalues exceeded the first but not second eigenvalues that emerged from parallel analysis (respectively, 1.40 and 1.27), suggesting a clear one-factor solution (See Table I).

In the pre-clinic data, the eigenvalues (and associated \% of variances) were $3.87(42.9 \%), 1.25$ (13.9\%), $0.82(9.1 \%), 0.81$ (9.0\%), 0.66 (7.4\%), 0.51 (5.6\%), 0.43 (4.8\%), $0.36(3.9 \%)$, and $0.28(3.1 \%)$. Parallel analysis suggested a two-factor solution. However, the second factor was weak (accounting for only $13.9 \%$ of the variance) and following oblique rotation had an eigenvalue of 0.70 , suggesting the factor had less explanatory value than any individual scale item. The weakness of the factor, combined with the result of the post-clinic data, suggests that the scale is best conceived as unifactorial. Based on this, we computed a single overall PPC score, which was used in all subsequent analyses.

Internal consistency was assessed for total PPC scores in the pre- and post-clinic datasets $(n=395,241$, respectively), and was found to be high (Cronbach's $\alpha=0.83$ in both datasets). We explored the relationships between PPC and age, gender, ethnicity, condition type, reason for referral, and whether patients described themselves as active in a patient support group at baseline $(n=395)$. There were no significant correlations with PPC, thus ruling out background influences. As none were significant, we present correlations below without partialing out demographic factors. 
TABLE I. Factor Loadings

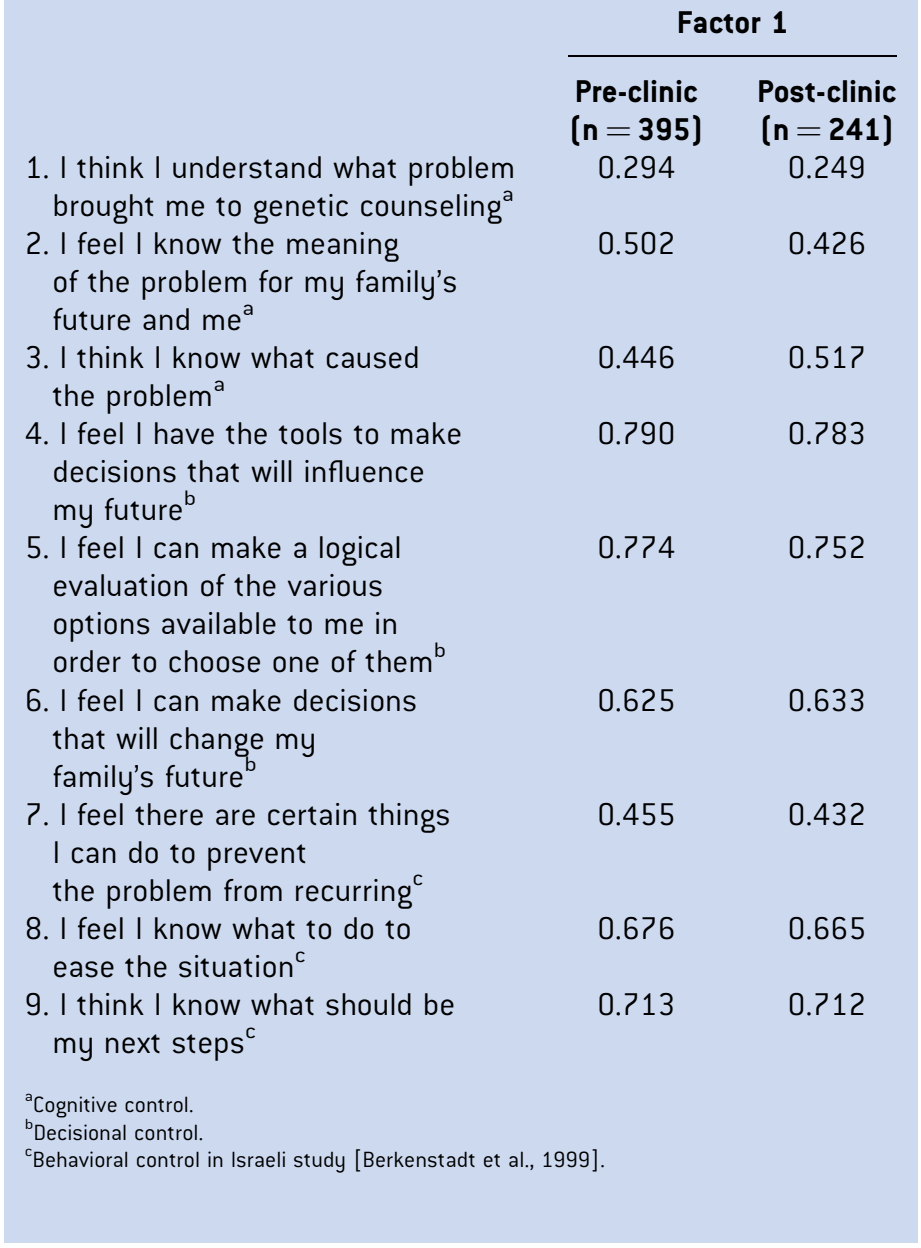

\section{Convergent Validity}

To test the expected relationships between PPC and IHLC, SWL, anxiety, depression, and authenticity, we correlated total PPC scores with measures of these constructs in the pre-clinic sample $(\mathrm{n}=395)$. As expected, PPC was significantly positively correlated with IHLC $(\mathrm{r}=.350, P<0.01)$, SWL $(\mathrm{r}=.333, P<0.01)$, and authenticity $(\mathrm{r}=.150, P<0.05)$, with the highest correlation being with IHLC. These findings provide some evidence of convergent validity. As expected, PPC was significantly negatively correlated with depression $(\mathrm{r}=-0.168, P<0.01)$, providing further evidence of convergent validity. PPC was not significantly correlated with anxiety ( $\mathrm{r}=-0.110$, not significant), which was unexpected.

Change in PPC scores following attendance at clinic $(\mathrm{N}=241)$ was significantly positively correlated with satisfaction $(\mathrm{r}=.322$, $P<0.01$ ), as measured by the short form SWGC scale. This relationship had been predicted, and provides further evidence of construct validity.

\section{Discriminant Validity}

As expected, PPC was not significantly correlated with either FHLC $(r=-0.094$, not significant), or PHLC $(r=-0.034$, not significant), providing evidence of discriminant validity.

\section{Known Groups Validity/Sensitivity to Change}

To further explore construct validity, we compared total PPC scores before and after clinic attendance using a paired samples $t$-test $(\mathrm{n}=241)$. PPC levels were significantly higher following clinic attendance [pre-clinic: Mean $(\mathrm{SD})=8.71$ (3.95); post-clinic: Mean $(\mathrm{SD})=10.31(3.87) ; \mathrm{t}=6.704, P<0.001]$. This provides evidence of both sensitivity to change of the PPC, which was able to detect a small-to-medium sized increase in PPC (Cohen's $\mathrm{d}=0.40$ ), and of known groups validity.

\section{DISCUSSION}

The present study reports the psychometric properties of the English translation of the PPC when used with a sample of clinical genetics patients in the UK. This is the first validation of the English translation of the PPC and suggests it has good psychometric properties. The PPC was shown to have good internal consistency, convergent validity, discriminant validity, and sensitivity to change. The PPC can detect significantly elevated levels of PPC following attendance at a genetics clinic, with a small-to-medium effect size. This also served as one test of known groups validity, as it was expected that levels of PPC would be higher in the post-clinic group than in the pre-clinic group. In addition, post-clinic PPC is significantly correlated with SWGC.

This study confirms that the English translation of the PPC could be useful as a measure of outcome from using clinical genetics services, and we know from previous research that PPC is valued as an outcome by patients of clinical genetics services, and by genetics clinicians in the UK [Payne et al., 2007]. This study adds to previous research by demonstrating that the PPC is sensitive to change in three different languages, cultures and health settings, providing further evidence that PPC is a measurable patient outcome from using clinical genetics services. It is well-recognized that high PPC reflects good psychological functioning [Skinner, 1996], so it is likely that increased PPC following attendance at a genetics clinic is a reflection that the goals of genetic counseling, which include facilitating adaptation in patients [Fraser, 1975; Biesecker and Peters, 2001], are being achieved to some degree.

As expected, PPC scores were (a) significantly positively correlated with IHLC, SWL and Authenticity, and (b) significantly negatively correlated with depression. Change in PPC scores following attendance at a genetics clinic was significantly positively correlated with SWGC. These significant correlations are evidence of convergent validity of the PPC because PPC scores correlated with scores on other instruments in the theoretically expected direction. Furthermore, also as expected, PPC was not correlated with either PHLC or FHLC scores, providing evidence of discriminant validity of the instrument. Together these findings establish construct validity of the PPC.

The finding in this study that PPC was significantly correlated with depression, but not with anxiety, was unexpected, because anxiety and depression are often treated together under the general umbrella of psychological distress. This approach has enabled rapid measurement of distress in hospital, outpatient, community, and research settings [Zigmond and Snaith, 1983; Herrmann, 1997]. However, anxiety and depression are in fact separate, though 
related constructs [Clark and Watson, 1991; Snaith, 1993; Dunbar et al., 2000; Caci et al., 2003], which may reflect different coping mechanisms [Hong, 2007]. The differential associations between PPC and anxiety/depression contribute to a clearer understanding of the PPC construct. Our findings concur with predictions of the helplessness/hopelessness model of depression, which maintains that the causes of depression are located in perceptions that one has no control over stressful life events, and that perceived control would correlate with depression better than anxiety [Abrahamson et al., 1978; Alloy et al., 1990 ], which is what we found. Therefore, we interpret the findings as further evidence of construct validity of the PPC.

An important strength of this research is the good sample sizes achieved, both pre- and post-clinic. However, only $61 \%$ of those completing questionnaires pre-clinic, also completed a questionnaire post-clinic. It is possible that the post-clinic sample is biased toward those who benefited from attendance, and may have resulted in an overestimation of the effect size. In addition, participation in the study was voluntary, and the participation rate achieved was relatively low $(32.8 \%)$. The low response rate may have been because the questionnaire pack and request to participate in the study were not sent to patients with their genetics clinic appointment, but rather were sent by the responsible genetics clinician (unknown to the patients at the time) in a separate mailing. This was a requirement of ethical approval for the study. Those who chose to participate therefore may not represent all patients.

A further limitation of this study is that the sample was overrepresented by (1) people describing themselves as female and White British (2) patients referred for cancer risks (with only small numbers of patients in other categories, e.g., neurological/neuromuscular conditions, chromosome abnormalities, and genetic eye conditions) [McAllister et al., 2011]. In addition, the sample was under-represented by patients referred for reproductive risks and predictive genetic testing. We could not determine whether this reflected the profile of referrals to the clinic, as ethical constraints prevented us from collecting data from the clinical records of patients who declined to participate. Furthermore, although $43 \%$ of participants were offered a genetic test in clinic, none of these had the result of this test at the time they completed their post-clinic questionnaire. This is likely to have influenced the effect size estimation.

The original PPC measure was developed using Averill's tripartite structure of PPC (behavioral, decisional, and cognitive control) [Averill, 1973]. The present study suggests that the English translation of the PPC questionnaire has one factor, consistent with findings regarding the Dutch translation [Smets et al., 2006], the only other published research using EFA. These findings do not contradict Averill's tripartite specification of PPC, but simply suggest that for patients involved in genetic counseling, behavioral, decisional, and cognitive control normally co-occur, so that they form a unitary appraisal of PPC. This suggests that the English translation of the PPC scale should be given a single overall score.

We conclude that the English translation of the PPC has good psychometric properties when used as a one-dimensional scale, using a simple sum of the items for analyses, and has potential as a useful measure of outcome for clinical genetics services. As with all psychometric instruments, the evidence for the psychometric properties and usefulness of the PPC for evaluating clinical genetics services will be strengthened by further research. In particular, it will be important to (1) test the psychometric properties of the English translation of the PPC with North American and Australian samples, (2) conduct long-term follow-up studies, and (3) explore the properties of the English translation of the PPC in different kinds of genetic counseling samples, in particular reproductive and non-cancer genetic counseling samples, and with samples of patients undergoing diagnostic and predictive genetic testing (including further tests of known-groups validity), and (4) compare PPC scores with newer measures of satisfaction with clinical genetics [e.g., Zellerino et al., 2009].

\section{ACKNOWLEDGMENTS}

The authors particularly thank all the participating patients who completed questionnaires. They also thank all the clinicians in Genetic Medicine, St. Mary's Hospital, Manchester for recruiting their patients for this study. This research was funded by the Medical Research Council, and supported by the NIHR Manchester Biomedical Research Centre. The views expressed in this article are those of the authors, and not those of the funding body. Ethical opinions were provided by the North West 7 NHS Research Ethics Committee (Ref. 09/H1008/111).

\section{REFERENCES}

Abrahamson LY, Seligman MEP, Teasdale JD. 1978. Learned helplessness in humans: Critique and reformulation. J Abnorm Psychol 87:49-74.

Alloy LB, Kelly KA, Mineka S, Clements CMI. 1990. Comorbidity of anxiety and depressive disorders: A helplessness-hopelessness perspective. In: Coninger JDMCR, editors. Comorbidity of mood and anxiety disorders. Washington, DC: American Psychiatric Press. pp 499-543.

Averill JR. 1973. Percieved control over aversive stimuli and its relationship to stress. Psychol Bull 80:286-303.

Berkenstadt M, Shiloh S, Barkai G, Bat-Miriam-Katznelson M, Goldman B. 1999. Percieved personal control (PPC): A new concept in measuring outcome of genetic counseling. Am J Med Genet 82:53-59.

Biesecker BB, Peters K. 2001. Process studies in genetic counseling: Peering into the black box. Am J Med Genet 106:191-198.

Caci H, Bayle FJ, Mattei V, Dossios C, Robert P, Boyer P. 2003. How does the Hospital Anxiety and Depression Scale measure anxiety and depression in healthy subjects? J Psychiatr Res 118:89-99.

Clark LA, Watson D. 1991. Tripartite model of anxiety and depression: Psychometric evidence and taxonomic implications. J Abn Psychol 100:316-336.

Davey A, Rostant K, Harrop K, Goldblatt J, O’Leary P. 2005. Evaluating genetic counselling: Client expectations, psychological adjustment and satisfaction with service. J Genet Couns 14:197-206.

Dunbar M, Ford G, Hunt K, Der G. 2000. A confirmatory factor analysis of the Hospital Anxiety and Depression Scale: Comparing empirically and theoretically derived structures. Br J Clin Psychol 39:79-94.

Fabrigar LR, Wegener DT, MacCallum RC, Strahan EJ. 1999. Evaluating the use of exploratory factor analysis in psychological research. Psychol Methods 4:272-299.

Fraser FC. 1975. Genetic counseling. Am J Hum Genet 27:240-242. 
Gilbody S, Richards D, Barkham M. 2007. Diagnosing depression in primary care using self-completion instruments: UK validation of PHQ-9 and CORE-OM. Br J Gen Pract 57:650-652.

Herrmann C. 1997. International experiences with the Hospital Anxiety and Depression Scale-a review of validation data and clinical results. J Psychosom Res 42:17-41IBM. SPSS Version 16.0. Chicago, Illinois.

Hong RY. 2007. Worry and rumination: Differential associations with anxious and depressive symptoms and coping behaviour. Behav Res Ther 45:277-290.

Marteau M, Bekker H. 1992. The development of a six-item short-form of the state scale of the Spielberger State-Trait Anxiety Inventory (STAI). Br J Clin Psychol 31:301-306.

McAllister M. 2010. Outcome measures in clinical genetics services. In: Kristoffersson U, Schmidtke J, Cassiman J-J, editors. Quality issues in clinical genetics services. Dordrecht, Heidelberg, London, New York: Springer. pp 183-190.

McAllister M, Wood A, Dunn G, Shiloh S, Todd C. 2011. The Genetic Counseling Outcome Scale: A new patient-reported outcome measure for clinical genetics services. Clin Genet 79:413-424.

Morriss J, Coyle D. 1994. Quality of life questionnaires in cancer clinical trials: Imputing missing values. Psychooncology 3:215-222.

Pavot W, Diener E. 1993. Review of the satisfaction with life scale. Psychol Assess 5:164-172.

Payne K, Nicholls SG, McAllister M, MacLeod R, Ellis I, Donnai D, Davies LM. 2007. Outcome measures for clinical genetics services: A comparison of genetics healthcare professionals and patients' views. Health Policy 84:112-122.

Pieterse AH, Ausems MGEM, Spreeuwenberg P, van Dulmen S. 2011. Longer-term influence of breast cancer genetic counseling on cognitions and distress: Smaller benefits for affected versus unaffected women. Pat Ed Counsel Epub, 10 Feb 2011: DOI:10.1016/j.pec.2011.01.017.

Shiloh S, Avdor O, Goodman RM. 1990. Satisfaction with genetic counseling: Dimensions and measurement. Am J Med Genet 37:522-529.

Shiloh S, Berkenstadt M, Meiran N, Bat-Miriam-Katznelson M, Goldman B. 1997. Mediating effects of Percieved Personal Control in coping with a health threat: The case of genetic counselling. J Appl Soc Psychol 27:1146-1174.

Skinner E. 1996. A guide to constructs of control. J Pers Soc Psychol 71:549-570.

Smets EMA, Pieterse AH, Aalfs CM, Ausems MGEM, van Dulman AM. 2006. The Percieved Personal Control (PPC) Questionnaire as an outcome of genetic counseling: Reliability and validity of the instrument. Am J Med Genet Part A 140A:843-850.

Snaith P. 1993. What do depression rating scales measure? Br J Psychiatr 163:293-298.

Wallston KA, Wallston BS, DeVellis R. 1978. Development of the multidimensional health locus of control scales. Health Edu Monogr $6: 160-170$.

Wood AM, Linley PA, Maltby J, Baliousis M, Joseph S. 2008. The authentic personality; a theoretical and empirical conceptualization and the development of the Authenticity scale. J Couns Psychol 55:385-399.

Zellerino B, Milligan SA, Brooks R, Freedenberg DL, Collingridge DS, Williams MS. 2009. Development, testing and validation of a patient satisfaction questionnaire for use in the clinical genetics setting. Am J Med Genet Part C 151C:191-198.

Zigmond AS, Snaith RP. 1983. The Hospital Anxiety and Depression Scale. Acta Psychiatr Scand 67:361-370.

Zwick WR, Velicer WF. 1986. Comparison of five rules for determining the number of components to retain. Psychol Bull 99:432-442. 